\title{
Modélisation des propriétés diélectriques des nanocomposites
}

Mohamed Essaid ACHOUR ${ }^{1^{*}}$, Aziz DROUSSI', Fouad LAHJOMRI ${ }^{2}$ et Amane OUERIAGLI ${ }^{3}$

${ }^{\prime}$ Equipe Systèmes, Propagation et Caractérisation des Matériaux (LASTID), Département de Physique, Faculté des Sciences, B.P.133, 14000 Kénitra, Maroc

${ }^{2}$ Laboratoire des Technologies de l'Information et de la Communication, Ecole Nationale des Sciences Appliquées de Tanger (ENSAT) B.P. 1818,

Tanger Principale, Maroc

${ }^{3}$ Laboratoire de Physique des Solides et des Couches Minces, Faculté des Sciences, Sémlalia, Marrakech, Maroc

*Correspondance,courriel:sachoum@yahoo.fr

\section{Résumé}

Dans ce travail, nous nous intéressons aux propriétés diélectriques dans le domaine des micro-ondes $(2.45,9.50$ et $35 \mathrm{GHz})$ des composites constitués des inclusions de noir de carbone dans une matrice résine époxyde. Nous avons utilisé deux types de carbone : le monarch 700 et le sterling, dont les diamètres des inclusions sont respectivement 0.018 et $0.175 \mu \mathrm{m}$. Les résultats obtenus montrent que la taille des particules conductrices parait être un paramètre prépondérant pour définir la permittivité complexe de ces composites. II résulte de cette étude que les modèles proposés pour la détermination de permittivité de ces milieux, devront nécessairement prendre en considération la taille des inclusions.

Mots-clés : Lois de mélange, propriétés diélectriques, permittivité complexe, nanocomposites, matrice époxyde, inclusions de carbone.

\section{Abstract}

\section{Modeling of the dielectric properties of nanocomposites}

In this work, we are interested in the dielectric properties in the microwave (2.45, 9.50 and $35 \mathrm{GHz}$ ) of composites consisting inclusions of carbon black in an the epoxy 
matrix. We used two types of carbon: the monarch 700 and sterling, whose diameters inclusions are 0,018 and $0,175^{\mu m}$, respectively. The results show that the particle size of conducting particles seems to be a dominant parameter in defining the complex permittivity of these composites. It is clear from this study that the proposed models for the determination of permittivity of these materials, must necessarily take into account the size of the inclusions.

Keywords : Mixture laws, dielectric properties, complex permittivity, nanocomposites, epoxy matrix, carbon inclusions

\section{Introduction}

Les matériaux composites sont couramment utilisés dans l'industrie (automobile, bâtiment, matériel électrique, médicales,...). II en résulte une forte demande de matériaux diélectriques de propriétés bien définies, matériaux transparents aux ondes électromagnétiques ou au contraire matériaux absorbants. Or les propriétés des matériaux naturels ne satisfont généralement pas aux exigences de l'industrie, en particulier pour les matériaux absorbants. Des matériaux artificiels, constitués d'une matrice thermodurcissable (résine polyester, phénolique, époxyde,..) et de renfort (fibres de verre, carbone ou Kevlar,..) ont alors été utilisés dans l'objectif de répondre à ces besoins. Les propriétés électromagnétiques (permittivité, conductivité électrique) des constituants dépendent des propriétés des constituants notamment de leurs concentrations volumiques [1-4]. Une modification des concentrations entraîne alors une modification des propriétés du mélange, ce qui permet de les ajuster à la demande. Le problème consiste alors de définir un mélange qui aura une permittivité et une conductivité connues à l'avance.

Dans cet article, nous nous intéressons à l'étude de la réponse diélectrique dans le domaine des micro-ondes des échantillons formés des inclusions : noirs de carbone, et d'aluminium, dispersés dans une matrice époxyde de type DGEBA (diglycidyléther du bisphénol A). Nous avons utilisé deux types de carbone : le monarch 700 et le sterling, dont les diamètres des inclusions sont respectivement 0.018 et $0.175 \mu \mathrm{m}$. Les résultats de l'ensemble des mesures effectuées sont analysés en fonction de la concentration volumique du milieu conducteur et aussi en fonction de la taille des inclusions. 


\section{Matériel et méthodes}

\section{2-1. Matériaux composites étudiés}

Les particules conductrices sont dispersées dans la résine avec durcisseur de conductivité électrique $\sigma_{D C} \approx 10^{-15}(\Omega . \mathrm{cm})^{-1}$ et de masse volumique $\rho=$ $1.16\left(\mathrm{~g}^{\mathrm{cm}} \mathrm{cm}^{-3}\right)$. Le mélange est réalisé avec soin de façon à obtenir une répartition uniforme des particules dans la matrice résine. Après une polymérisation d'environ de $24 \mathrm{~h}$ à la température ambiante, le milieu est solide. La technique de préparation est donnée en détail dans la référence [5].

\section{2-2. Techniques de mesure}

Les mesures de la permittivité complexe $\varepsilon^{*}=\varepsilon^{\prime}{ }_{-} \varepsilon^{\prime}{ }^{\prime \prime}$ aux fréquences 2.45 et 9.50 $\mathrm{GHz}$ sont effectuées par des cavités résonantes. Cette technique consiste à déterminer expérimentalement la variation de la fréquence de résonance et du facteur surtension du à l'introduction de l'échantillon dans la cavité résonante. La méthode de petites perturbations permet de relier la permittivité complexe $\varepsilon^{*}$ du matériau aux variations de la fréquence et du facteur de surtension [6]. Les mesures de $\varepsilon^{*}$ en $35 \mathrm{GHz}$ sont réalisées à l'aide d'un pont d'impédances [7].

\section{Résultats}

Les variations de $\varepsilon^{\prime}$ et $\varepsilon^{\prime \prime}$ en fonction de la concentration volumique des inclusions $\phi$ pour les séries monarch 700 - époxyde et sterling - époxyde, aux fréquences 9.5 et $35 \mathrm{GHz}$, sont données dans les Figures $\mathbf{l - a}$ et $\mathbf{l - b}$. On remarque que l'évolution des permittivités $\varepsilon^{\prime}$ et $\varepsilon^{\prime \prime}$ de nos échantillons en fonction de la concentration volumique $\phi$ en carbone est une progression continue, contrairement à ce que l'on observe en basses fréquences où il y'a une divergence au voisinage du seuil. Ces mesures démontrent que la théorie de la percolation électrique ne s'applique pas en hautes fréquences et dans le domaine des micro-ondes. 
En hautes fréquences et en micro-ondes, la théorie de percolation n'est pas applicable; en effet dans ces domaines de fréquence, les champs électromagnétiques sont rejetés hors du milieu conducteur, la propagation s'effectue dans la matrice diélectrique. Par ailleurs, pour modéliser le comportement diélectrique à la fréquence de $2.45 \mathrm{GHz}$, nous avons utilisé les lois de mélange suivantes [8]:
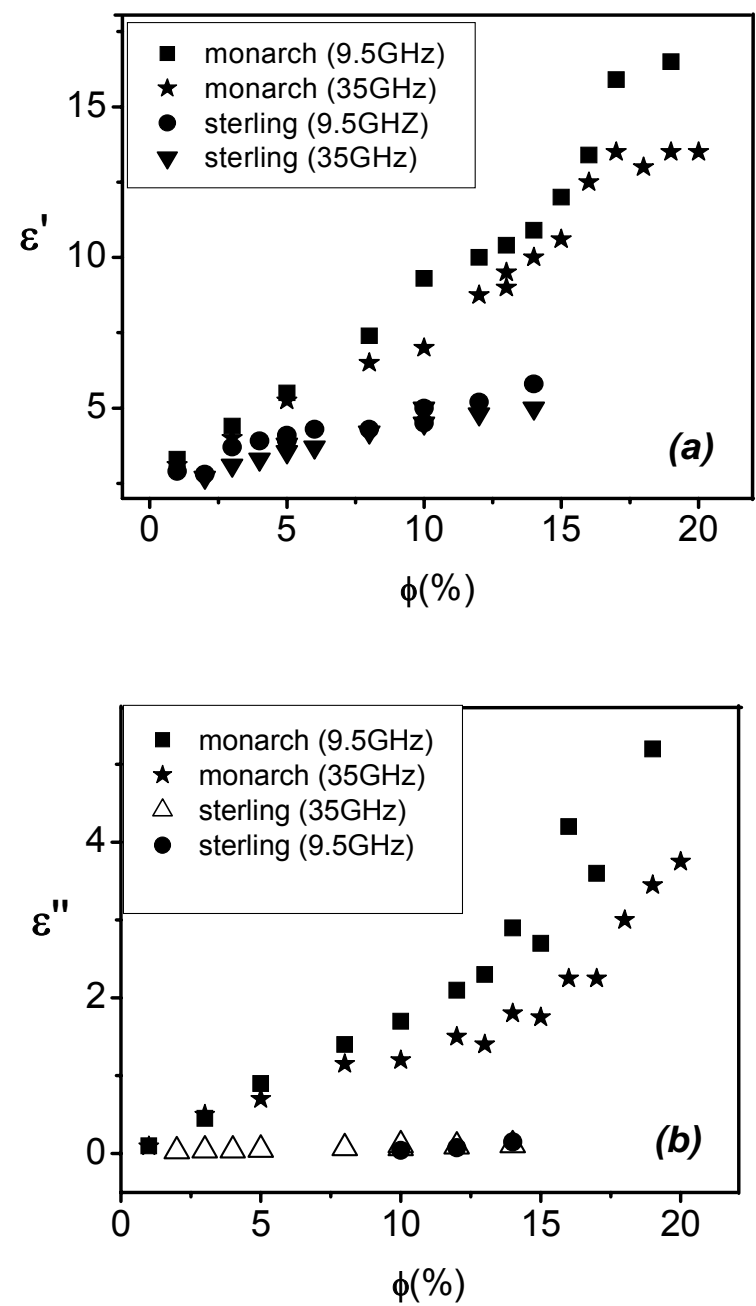

Figure 1 : Variation de la permittivité complexe des composites monarch-Epoxyde et sterling-Epoxyde en fonction de la concentration ${ }^{\phi}$ en carbone dans le domaine des microondes : partie réelle (a) et partie imaginaire de la permittivité complexe 
- loi de Maxwell-Garnet (MG) [9]:

$$
\varepsilon^{*}=\varepsilon_{m}^{*} \frac{\varepsilon_{c}^{*}(1+2 \phi)+2 \varepsilon_{m}^{*}(1-\phi)}{\varepsilon_{c}^{*}(1-\phi)+\varepsilon_{m}^{*}(2+\phi)}
$$

- loi de Bruggeman (BR) [10]:

$$
\varepsilon^{*}=\varepsilon_{m}^{*} \frac{\varepsilon_{c}^{*}(1+2 \phi)+2 \varepsilon_{m}^{*}(1-\phi)}{\varepsilon_{c}^{*}(1-\phi)+\varepsilon_{m}^{*}(2+\phi)}
$$

-loi de Bottcher (BT) [11] :

$$
\frac{\varepsilon^{*}-\varepsilon_{m}^{*}}{3 . \varepsilon^{*}}=\phi \cdot \frac{\varepsilon_{c}^{*}-\varepsilon_{m}^{*}}{\varepsilon_{c}^{*}+2 . \varepsilon_{m}^{*}}
$$

- loi de Looyenga (LG) [12]:

$$
\left(\varepsilon^{*}\right)^{1 / 3}=\phi\left(\varepsilon_{c}^{*}\right)^{1 / 3}+(1-\phi)\left(\varepsilon_{m}^{*}\right)^{1 / 3}
$$

- loi de Lichtenecker et Rother (LR) [13]:

$$
\left(\varepsilon^{*}\right)^{k}=\phi\left(\varepsilon_{c}^{*}\right)^{k}+(1-\phi)\left(\varepsilon_{m}^{*}\right)^{k}
$$

où $\varepsilon^{*}=\varepsilon^{\prime}-j \varepsilon^{\prime \prime}$ est la permittivité complexe du milieu hétérogène, $\varepsilon_{m}^{*}=\varepsilon_{m}^{\prime}-j \varepsilon_{m}^{\prime \prime}$ est la permittivité de la matrice et $\varepsilon_{c}^{*}=\varepsilon_{c}^{\prime}-j \varepsilon_{c}^{\prime \prime}$ la permittivité des inclusions conductrices. Le terme $\phi$ est la concentration volumique des inclusions. Nous avons reporté sur les figures 3 et 4 , les variations de $\varepsilon^{\prime}$ et $\varepsilon^{\prime \prime}$, calculées à l'aide des expressions (1) à (5) des composites en fonction de la concentration volumique en noir de carbone, à $2.45 \mathrm{GHz}$, avec les permittivités de la résine époxyde : $\varepsilon_{m}{ }^{*}=3.4-j 1.05$ et celle du carbone: ${ }^{*}{ }^{*}=60-j 20$ [14]. Signalons que pour des concentrations aussi petits, toutes les lois de milieu effectif dérivant de la formule de Clausius-Mossotti se confondent à leur développement asymptotique commun à $p \rightarrow 0$ et ne rendent aucunement compte des données expérimentales. En revanche la loi de Lichtenecker, autorise une variation forte selon le paramètre ajustable $k$. La loi de Lichtenecker dérive d'un argument d'homogénéité mathématique sur les mélanges. Les variations de $\varepsilon^{\prime}$ expérimentales et celles calculées par l'équation de Lichtenecker pour $k=1.2$ sont 
presque confondues. D'après la Figure 2, nous constatons que dans le cas du mélange carbone (monarch 700)- époxyde, la loi de Lichtenecker (avec $k=1.2$ ) est en accord avec les valeurs expérimentales pour $\varepsilon^{\prime}$. Par contre, pour $\varepsilon^{\prime \prime}$ les valeurs expérimentales sont en désaccord avec les courbes calculées figure 3 (b). Ce désaccord persiste à partir de $\phi=10-15 \%$.
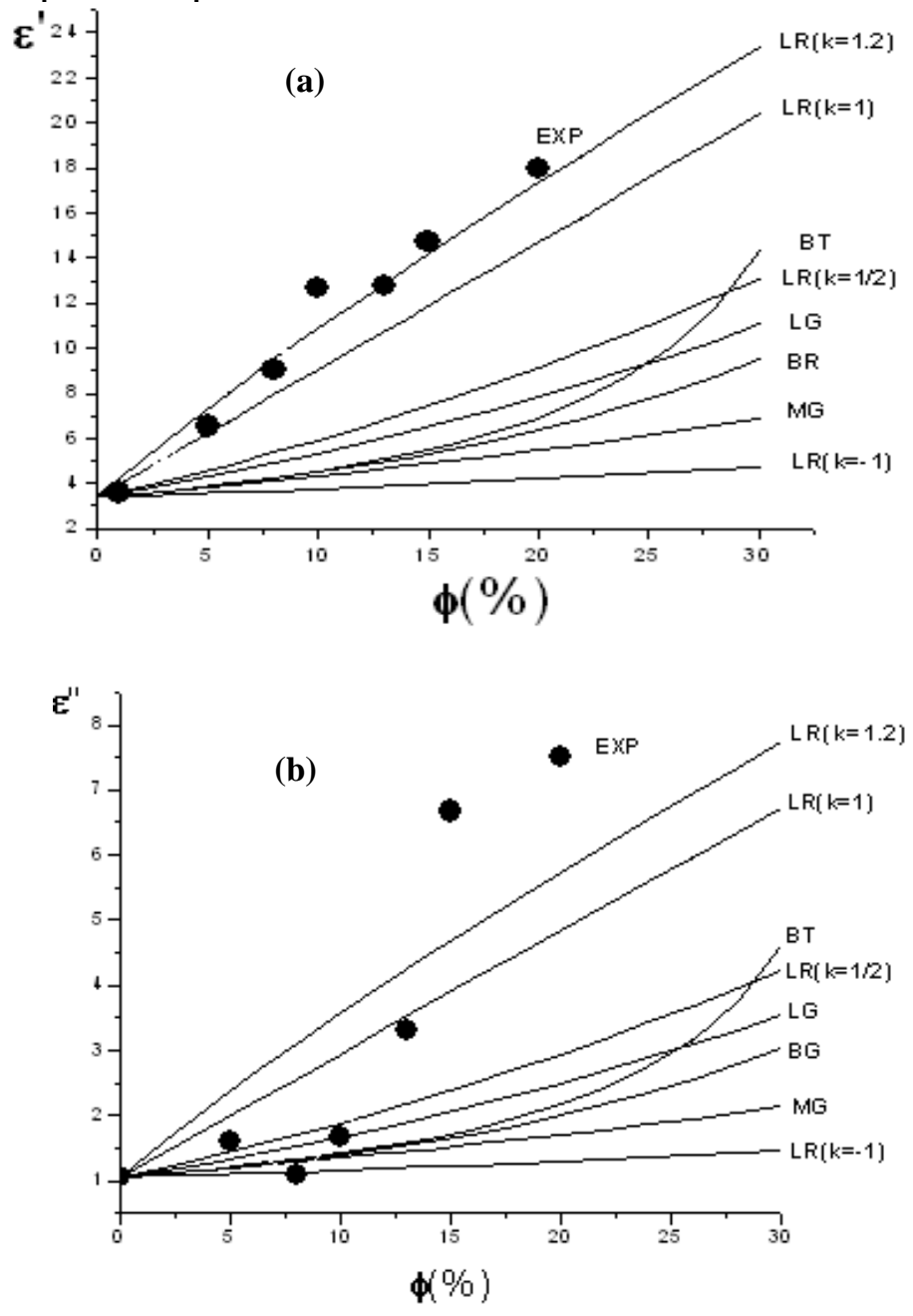

Figure 2 : Variation de $\varepsilon^{\prime}$ (a) et de $\varepsilon^{\prime \prime}$ (b) de composites Monarch 700-Epoxy en fonction de la concentration volumique $\phi$ en noirs de carbone à 2.45 $\mathrm{GHz}$. lesvaleurs théoriques sont calculées par les différentes lois de mélanges avec: ${ }^{\varepsilon_{c}}{ }^{*}=60-j 20$ ef $^{\varepsilon_{m}}{ }^{*}=3.4-j 1.05$ 
Dans cette étude, on a utilisé quelques lois de mélange conçues pour les propriétés diélectriques complexes du mélange diélectrique -diélectrique où la phase conductrice est traitée comme un matériau diélectrique avec de très fortes pertes. Ces lois de mélanges concernant le composite diélectrique - diélectrique peuvent être étendues aux matériaux à fortes pertes, pour tenir compte des inclusions conductrices; la permittivité complexe du mélange se calcule en supposant que la permittivité des inclusions conductrices est infinie [8]. Ces lois donnent des résultats assez satisfaisants pour des milieux pour lesquels les constituants sont de même nature : mélanges de diélectriques à faibles pertes ou de conducteurs [15-16]. Le désaccord devient considérable quand les deux constituants sont de nature différente : isolant - conducteur. En effet, les lois sont établies en admettant que les particules appartenant aux différents constituants, ont des formes sphériques. Dans la réalité les composites utilisés sont souvent des particules sphériques noyées dans une matrice continue. Les résultats expérimentaux obtenus montrent que les propriétés diélectriques de ces composites dépendent de la concentration et aussi de la taille des inclusions. II résulte de cela que les modèles proposés devront nécessairement tenir compte de la géométrie des inclusions et plus particulièrement de leur taille. . La multitude des lois de mélanges rencontrées dans la littérature reflète ces difficultés. Des travaux récents ont montré que, pour des milieux où les concentrations d'inclusions sont importantes, le recours aux méthodes numériques s'avère indispensables.

\section{Conclusion}

Nous avons utilisé différentes lois de mélange pour décrire le comportement diélectrique de nos échantillons. L'utilisation de ces lois, dans leurs formes originales, n'a pas donné un accord satisfaisant. Ceci peut être dô au fait que plusieurs paramètres (tailles des inclusions, formation d'agrégats,...) ne sont pas pris en considération. En effet, certaines lois de mélange ont subi des modifications pour tenir compte de l'effet de la dimension des inclusions. Ce modèle, qui est valable lorsque le rayon des particules est au moins dix fois plus petit que la longueur d'onde, ne peut être comparé aux résultats de mesures dans le domaine des micro-ondes. 


\section{Références}

[1] - A. Mdarhri, C. Brosseau and F. Carmona, J. Appl. Phys., 101, 8 (2007) 084111084111-12.

[2] - V. Myroshnychenko and C. Brosseau. C Phys. Rev. E 71, 016701 (2005) 1- 16.

[3] - L.C. Costa, F. Henry, M.A. Valente, S.K. Mendiratta and A.S. Sombra, Euro. Polym., J. 38 (2002) 1495-1499.

[4] - M. El MALH I, M .E. ACHOUR, F.LAHJOMRI and Y. BENSALAH, J. Mater. Sci. Lett. 18 (1999) 613-616.

[5] - L. Salomé and F. Carmona, Carbon, 29 (1991) 599- 604

[6] - S. Li, C. Akyel and R. G. Bosisio, J. IEEE MIT, 29, 10, 230, (1981) 1041- 1048.

[7] - M. E. ACHOUR, M. El MALHI, J.L. MIANE, and F. CARMONA, J. Appl. Polym. Sci,, 61 (1996) 2009-2013.

[8] - M. E. AcHouR, M. El MaLHi, J.L.Miane, F. CARMONA and F. LAHromRi, J. Appl. Polym. Sci. , 73, (1999) 969-973.

[9] - J.C. Maxwell-Garnett, Philos Trans R Soc London, 203 (1904) 385-420

[10] - D. A. G . Bruggeman, Ann Phys., 24 (1935) 636- 679.

[11] - C. J. F. Böttcher, Theory of Electric Polarization; Elsevier: Amsterdam (1952) 415420.

[12] - H. Looyenga, Physica, 31 (1965) 401-404.

[13] - K. Lichtenecker and K. Rother, Phys. Z., 32 (1931) 255-260.

[14] - J.C. Martin, J.M. Fornies-Marquina and A.M. Bottreau, Mol. Phys, 101, 12, 2(2003) 1789-1793.

[15] - A. Kraszewski, J. Microwave Power, 12 (1977) 215-222.

[16] - R. Landauer, J. Appl. Phys., 23 (1952)779-784. 